\title{
Most practices provide safe dental care but good prevention varies, says CQC
}

\section{By Adrian O’Dowd}

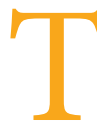

he vast majority of dental practices are providing safe, effective care but good prevention is much more variable across England, according to NHS regulator the Care Quality Commission

(CQC).

The CQC published its annual report State of Care 2017-18 on 11 October 2018 in which it assesses overall health and social care in England.

The regulator does not rate dental practices in the same way as GP practices or hospitals, but each year it inspects $10 \%$ of all registered primary care dental services in England.

The report showed $90 \%$ of dental practices inspected in the year to 31 March 2018 were meeting regulations and providing safe, effective care.

Inspection showed the majority of practices were following the advice of Public Health England (PHE) in Delivering better oral health ${ }^{2}$ meaning that people were receiving evidence-based advice on improving their oral health. However, this was not always extended to effective prevention, with a wide geographical variation across England of the number of children having teeth extracted (often in hospital under general anaesthetic) because of tooth decay.

There had been steady overall improvement in the oral health of children over many years thanks to dental practices providing preventative care, said the CQC, noting that in 2007-08, the average number of decayed, missing or filled teeth in five-year-olds was 1.11 and by 2016-17, it had dropped to 0.8 . However, there was wide variation at regional and local authority level, in both the severity and prevalence of dental decay.

An oral health survey carried out by PHE had found that this appears to be linked with levels of deprivation, as prevalence among the most deprived children was $33.7 \%$ and for the least deprived was $13.6 \%$.

CQC said the lowest average number of decayed, missing or filled teeth in five-year-olds was 0.1 in Waverley, Surrey, compared with 2.3 in Pendle in Lancashire. Other factors such as ethnicity, exposure to water fluoridation and geographic location were also independently associated with levels of dental decay in children.

Variation across the country was an overall theme of the report as the CQC said that while quality of health and social care had been largely maintained, and in some cases improved, from last year, people's experience of care varied depending on where they lived and how well different parts of local systems worked together.

Of the 1,336 dental practices inspected up to March 2018, the CQC said it had required 118 to take some action to improve and it took enforcement action against a further 17 practices. The main triggers of enforcement action were usually poor leadership cultures in practices, an unwillingness or inability to take prompt and decisive action to address issues, and a failure to act when things had gone wrong. A specific issue that the regulator had responded to swiftly was unsafe practice of conscious sedation of patients.

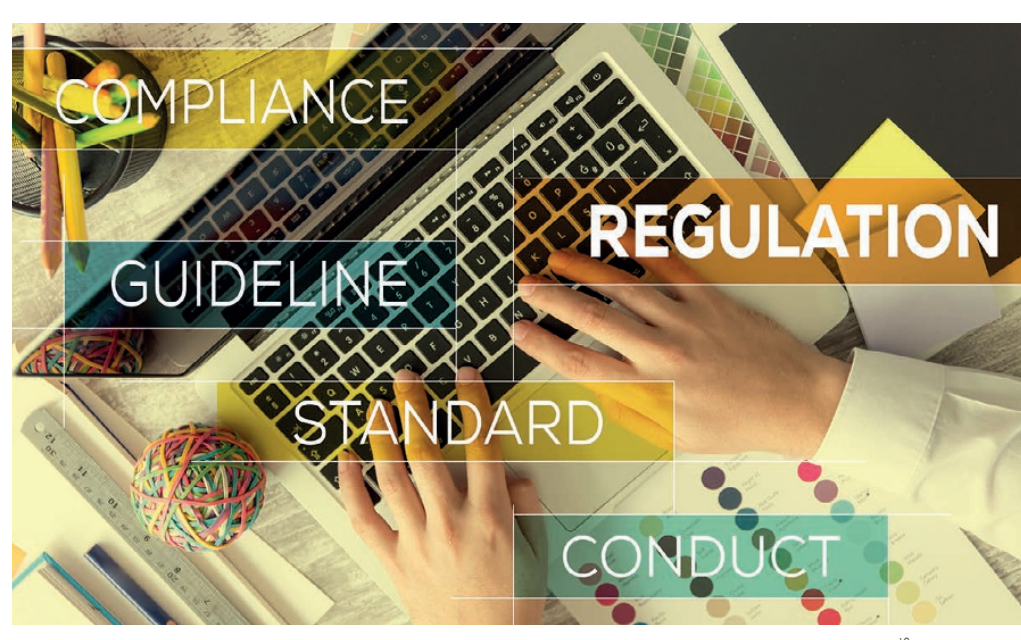

It had published reports in 2017-18 of the re-inspections of 182 dental practices and for $89 \%$ of these services, they were meeting regulations and no action was required.

Of the 16 practices where the CQC had taken enforcement action on its first inspection, 13 had improved on re-inspection so no action was taken. Of the 139 re-inspected practices where the CQC had initially applied requirement actions, $92 \%$ had addressed them and had no further actions.

However, on re-inspection it applied requirement actions for six of these practices, and a further five resulted in enforcement actions.

Lack of integration between dental practices and the rest of the healthcare sector was mentioned in the report.

The authors said: 'In the changing landscape of primary care, NHS dental practices are not fully involved in the developing models of integrated care.

'Within the dental sector itself there is consolidation as the size and number of corporate providers grows. Because dental practices are also able to choose how they balance NHS and private provision of treatment, this may affect people's access to NHS services in a local area.'

Ian Trenholm, CQC Chief Executive, said: 'This year's State of Care highlights both the resilience and the potential vulnerability of a health and care system where most people receive good care, but where access to this care increasingly depends on where in the country you live and how well your local health system works together. This is not so much a 'postcode lottery' as an 'integration lottery'.

'We've seen some examples of providers working together to give people joined-up care based on their individual needs. But until this happens everywhere, individual providers will increasingly struggle to cope with demand - with quality suffering as a result.

1. Care Quality Commission. The state of health care and adult social care in England 2017-18 (11 October 2018). Available at https://www.cqc.org.uk/publications/major-report/state-care (accessed 15 October 2018).

2. Public Health England. Delivering better oral health (12 June 2014). Available at https://www. gov.uk/government/publications/delivering-better-oral-health-an-evidence-based-toolkit-forprevention (accessed 15 October 2018). 